\title{
Telemedicine and Diabetic Retinopathy: Review of Published Screening Programs
}

\author{
Kevin Tozer, Maria A. Woodward and Paula A. Newman-Casey*
}

Department of Ophthalmology \& Visual Sciences, University of Michigan Medical School, Ann Arbor, Michigan 48105, USA

Received: September 05, 2015; Accepted: September 25, 2015; Published: October 11, 2015

*Corresponding author: Paula Anne Newman-Casey MD MS, Department of Ophthalmology \& Visual Sciences, University of Michigan Medical School, Ann Arbor, Michigan 48105, USA, E-mail: panewman@med.umich.edu

\begin{abstract}
Background: Diabetic Retinopathy (DR) is a leading cause of blindness worldwide even though successful treatments exist. Improving screening and treatment could avoid many cases of vision loss. However, due to an increasing prevalence of diabetes, traditional in-person screening for DR for every diabetic patient is not feasible. Telemedicine is one viable solution to provide high-quality and efficient screening to large number of diabetic patients.

Purpose: To provide a narrative review of large DR telemedicine screening programs.

Methods: Articles were identified through a comprehensive search of the English-language literature published between 2000 and 2014. Telemedicine screening programs were included for review if they had published data on at least 150 patients and had available validation studies supporting their model. Screening programs were then categorized according to their American Telemedicine Association Validation Level.

Results: Seven programs from the US and abroad were identified and included in the review. Three programs were Category 1 programs (Ophdiat, EyePacs, and Digiscope), two were Category 2 programs (Eye Check, NHS Diabetic Eye Screening Program), and two were Category 3 programs (Joslin Vision Network, Alberta Screening Program). No program was identified that claimed category 4 status. Programs ranged from community or city level programs to large nationwide programs including millions of individuals. The programs demonstrated a high level of clinical accuracy in screening for DR. There was no consensus amongst the programs regarding the need for dilation, need for stereoscopic images, or the level of training for approved image graders.
\end{abstract}

Conclusion: Telemedicine programs have been clinically validated and successfully implemented across the globe. They can provide a high-level of clinical accuracy for screening for DR while improving patient access in a cost-effective and scalable manner.

Keywords: Diabetic retinopathy; Telemedicine; Screening programs

\section{Introduction}

Diabetic Retinopathy (DR) represents a major national and international public health problem. DR is the most common cause of vision loss among working-age adults in the US [1]. Fortunately, when identified early and treated, the risk of vision loss is cut in half [2]. However, according to the National Committee for Quality Assurance, our current health care system meets guidelines for diabetic screening only by $40-60 \%$ of the time [3,4]. With a growing population of diabetics [5] and with work shortages projected for ophthalmologists [6], innovative approaches to diabetic retinopathy assessments could prevent needless vision loss. One approach is to use tele-ophthalmology to aid in the evaluation process by: 1) increasing local access to screening for diabetic retinopathy and 2) risk stratifying patients with diabetic eye disease so that those who require treatment can be scheduled more efficiently [7-9]. The American Telemedicine Association (ATA) has described four categories which classify telemedicine programs [10]. In this review, our aim was to provide a narrative review of the literature surrounding the four different categories of DR screening programs in the US and around the world.

\section{Methods}

Initial articles were identified by performing a PubMed search using the following key words: "diabetic retinopathy" or "diabetes and eye disease" and at least one of the following "telemedicine", "telepathology", "remote consultation", "mobile health units", "telemed", "teleimage", "teleEye", "telesurveillance", "teleophthalmology", "tele-consultation", or "tele-retina". Additional inclusion criteria were articles published in English, human studies, and publication after the year 2000. The final search was conducted on December 4, 2014. With this search, 168 articles were identified. For inclusion in this narrative review, a program had to have published data on at least 150 patients and describe the process of ATA validation for their telemedicine program. The purpose of this review was to focus on telemedicine programs that have been implemented. By limiting our review to programs that had evaluated at least 150 patients, we excluded programs that were in the pilot testing-phase. All abstracts were reviewed and 30 articles were identified that directly discussed validation or design of an included diabetic screening program. The bibliographies of articles that were read were then reviewed 
to identify additional articles not captured in the initial literature search, and a total of 35 articles pertaining to specific programs were included.

\section{American Telemedicine Association: Teleoph- thalmology Practice Recommendations}

The ATA has classified tele-ophthalmology screening programs into four categories. These categories define the level of clinical validation for a program and define program goals (Table 1). The ATA guidelines use ETDRS $30^{\circ}$, stereo sevenstandard field, color $35 \mathrm{~mm}$ slides as the gold standard for diabetic retinopathy diagnoses and grading and comparing all tele-ophthalmology programs to this gold standard [10].

Category 1 validation is for programs with the lowest level of clinical validation. In a Category 1 program, imaging can distinguish between no disease, very mild DR and greater than mild DR. No further level of severity stratification is possible in these programs. Thus, treatment decisions cannot be made.

Category 2 validation requires a program to be able to rate the severity of DR, differentiating between sight- threatening and non-sight threatening disease. Sight-threatening DR is defined as the presence of Diabetic Macular Edema (DME), severe nonproliferative DR, or any proliferative DR. With a Category 2 telemedicine system, decisions can be made about which, the patients require urgent referral for treatment.

Telemedicine systems that have undergone Category 3 validation can stratify the severity of DR to a greater degree than Category 2 systems. Category 3 systems can identify defined levels of non-proliferative DR (mild, moderate, or severe), proliferative DR (either early or high-risk), and the presence or absence of DME. In this category, decisions about appropriate treatment and follow-up can be made with similar certainty to that of a dilated fundus examination by an ophthalmologist. This type of telemedicine system allows remote management of less severe cases of DR and efficient scheduling of vision-threatening cases for different types of treatment that patient may need.

Category 4 has the highest burden of validation. These systems achieve diagnostic accuracy matching or exceeding the gold standard ETDRS photos for identifying and defining DR and DME. A Category 4 program could replace clinical examination or ETDRS photos for clinical or research applications. To our knowledge, to date, no such systems have been created or evaluated.

The category classifications help define what is possible in clinical applications. For example, if the goal of a program is not only to identify patients with DR, but also to risk stratify according to whether or not vision-threatening disease is present, Category 2 validation is required. If the program seeks to make treatment and follow-up decisions, Category 3 validation is required. In this review, we will describe DR screening tele-ophthalmology programs that have been validated and implemented in Categories 1, 2 and 3.

\section{Current telemedicine screening programs}

\section{Category 1 programs:}

Ophdiat (Paris, France): The Ophdiat ${ }^{\circledast}$ network was created in 2004 to serve the area around Paris, France. As of 2009,

Table 1: Description of each category within the American Telemedicine Associations (ATA) levels of validations. The levels of DR that can be distinguished.

\begin{tabular}{|c|c|c|c|c|}
\hline \multicolumn{5}{|c|}{ American Telemedicine Associations(ATA) Levels of Clinical Validation } \\
\hline & Category 1 & Category 2 & Category 3 & Category 4 \\
\hline $\begin{array}{c}\text { Level of } \\
\text { categorization }\end{array}$ & $\begin{array}{ll}\text { - } & \text { No or minimal } \\
\text { DR } \\
\text { - } \\
\text { More than } \\
\text { minimal Dr }\end{array}$ & $\begin{array}{ll}\text { - } & \text { No or minimal } \\
\text { DR } \\
\text { - } \quad \text { Mild or } \\
\text { moderate DR } \\
\text { - Sight } \\
\text { threatening DR } \\
\text { or DME }\end{array}$ & $\begin{array}{ll}\text { - } & \text { No DR } \\
\text { - } & \text { Mild DR } \\
\text { - } & \text { Moderate DR } \\
\text { - } & \text { Severe non - } \\
& \text { proliferative DR } \\
\text { - } & \text { Early proliferative } \\
& \text { DR } \\
\text { - } & \text { High risk DR } \\
\text { - } & \text { DME }\end{array}$ & $\begin{array}{ll}- & \text { No DR } \\
- & \text { Mild DR } \\
- & \text { Moderate DR } \\
- & \text { Severe non - } \\
& \text { proliferative DR } \\
- & \text { Early proliferative DR } \\
- & \text { High risk DR } \\
\text { - } & \text { DME }\end{array}$ \\
\hline Capabilities & Screening & $\begin{array}{l}\text { Screening and Risk } \\
\text { stratification }\end{array}$ & $\begin{array}{l}\text { Screening, Risk stratification, } \\
\text { treatment recommendations }\end{array}$ & $\begin{array}{l}\text { Exceeds ETDRS seven field photos } \\
\text { in determining level of DR/DME. } \\
\text { Can replace ETDRS photos in } \\
\text { clinical/research programs }\end{array}$ \\
\hline \multirow{3}{*}{ Example Programs } & $\begin{array}{l}\text { Ophdiat (Paris, France) } \\
(12)\end{array}$ & $\begin{array}{l}\text { EyeCheck (Netherlands) } \\
(24)\end{array}$ & $\begin{array}{l}\text { Joslin Vision Network } \\
\text { (Massachusetts, USA) } \\
(8,41,42,43)\end{array}$ & \multirow{3}{*}{ None } \\
\hline & $\begin{array}{l}\text { EyePacs (California, USA) } \\
(17)\end{array}$ & $\begin{array}{l}\text { NHS Diabetic Eye Screening } \\
\text { program (United Kingdom) } \\
(31,32,33,34,35)\end{array}$ & $\begin{array}{l}\text { University of Alberta } \\
\text { (Alberta, Canada) }(46,47,9)\end{array}$ & \\
\hline & $\begin{array}{l}\text { Digiscope (Maryland, } \\
\text { USA) (22) }\end{array}$ & & & \\
\hline
\end{tabular}


seventeen hospitals, 11 primary healthcare centers and two prisons were participating in screening, with 14 ophthalmologists serving as graders [7]. The Ophdiat ${ }^{\circledR}$ network used two $45^{\circ}$ degree photos, one centered on the posterior pole and one on the optic disc. The photographs were taken by trained nurses or orthoptists. Dilation was used if the photographer felt the pupil size affected image quality. Compressed Joint Photographic Experts Group (JPEG) images were graded remotely by an ophthalmologist within two business days and reports were sent back to primary care providers, who communicated the results and follow-up recommendations to the patients. Images were graded for level of DR, presence or absence of DME, and any other incidental findings. Patients with no DR or mild DR were followed with repeat photographs in 1 year. Those patients with worse than mild DR, had a visit scheduled with an ophthalmologist [7].

The Ophdiat ${ }^{\circledR}$ network had screened over 38,000 patients as of 2009 [7]. Implementation of this telemedicine program had increased screening rates from $50 \%$ in 2001 to $72.4 \%$ in $2005(P<0.001)$ while decreasing the amount of time spent by ophthalmology staff by $60 \%$ per case of diagnosed DR [11]. The ophthalmologist read the photographs and examined any positive results in person. Even with the $20 \%$ increase in total patients screened, this still led to a $60 \%$ reduction in time spent by the ophthalmologist (reading and examining positive screens in person) per case diagnosed.

The Ophdiat ${ }^{\circledR}$ network cites a study from Massin [12] that compared non-mydriatic digital photography to standard sevenfield ETDRS photos as evidence for the validity of the program. In that study, a comparison of the two imaging modalities in 147 eyes found an agreement of between $92 \%$ and $100 \%$ for the diagnosis of moderately severe to severe diabetic retinopathy. In another analysis of the Ophdiat network, 59/456 (13\%) of patients evaluated through the telemedicine program were referred for full ophthalmologic examination. Twenty-three (39\%) were referred for DR and 36 (61\%) were referred for other ocular pathology or due to ungradable images [13].

Five percent of all images are randomly selected for re-grading by a second ophthalmologist to ensure internal consistency. With this approach, they have achieved a 92-99\% agreement rate between different graders. They attributed a low ungradable rate $<10 \%$ to a structured training and monitoring program for the photographers [14].

EyePACS (California, US): EyePACS is license-free webbased DR screening tool developed in 2001 designed for primarycare offices. EyePACS offered the software for free but provided training and image reading by an optometrist for a fee. If an outside grader was used, the grader had to undergo EyePACS certification [15]. The system was initially piloted in 2006 on 3,500 patients [16], and as of 2010, EyePACS has facilitated over 53,000 screening encounters for DR in California [17]. However, of the $15 \%$ of patients referred for follow-up care through EyePACS screening, only 30\% returned to their eye care provider for follow-up [17].

EyePACS imaging protocol used three non-stereo images of each fundus with selective dilation. It used a similar imaging protocol, with the exception of stereopsis, to the well-studied Joslin Vision Network imaging protocol (discussed below) and that was their rationale for forgoing validation of their protocol for diagnosis of DR. However, they did validate the program for evaluation of Clinically Significant Diabetic Macular Edema (CSDME, as defined in the ETDRS study [2]). They used the presence of hard exudates within one disc diameter of the fovea as a surrogate marker for CSDME, which had been shown to have a good sensitivity for CSDME in non-stereoscopic imaging [16]. In a study looking at the diagnosis of CSDME in 143 patients using the EyePACS system compared to in-person examination by an optometrist, the program was found to have sensitivity between $75 \%$ and $93.8 \%$ and specificity between $85.1 \%$ and $88.5 \%$ between multiple image graders [17].

Digiscope (Baltimore, US): The Digiscope is a mydriatic, monochromatic red-free camera that images the central 45$50^{\circ}$ utilizing a series of 10 overlapping photos. The Digiscope was designed for the use in primary care offices. Red-free images have been shown to have increased sensitivity for detecting DR by increasing contrast visibility for readers [18]. The DigiScope featured automated functions, including pupil alignment, autofocus, and automated image acquisition in order to be more user-friendly and facilitate use by a wide variety of paraprofessional staff in primary care physicians' offices [19]. All images taken in the primary care providers' offices were uploaded to a reading center for grading by certified readers. These certified graders had $91 \%$ agreement with an ophthalmologist in the decision to refer a patient for further treatment for DR in a study involving over 1,600 eyes [20].

In 2004, researchers performed a validation study of the DigiScope in 111 diabetic patients compared to 7-field ETDRS stereo color photos to determine if DR was present or absent and if $>$ mild DR was present. There were similar rates of gradable photos $(85 \%$ for Digiscope versus $88 \%$ for standard color photos) and excellent agreement for determination of presence or absence of any level or retinopathy (kappa $=0.96,95 \%$ confidence interval 0.87-1.00). There was also good agreement between readers in distinguishing between > mild DR and less severe cases (kappa $=0.83,95 \%$ confidence interval 0.64-0.98). As the DigiScope was intended for screening only, further risk stratification was not evaluated [21]. After greater than 100,000 screening exams both in the US and abroad [22], the Digiscope has facilitated an average referral rate for pathology of around $20 \%$ in patients with no known prior diabetic retinopathy [23], which is approximately equal to the rates of referral for pathology in other large tele-ophthalmology diabetic retinopathy programs $[8,12]$.

\section{Category 2 programs:}

Eyecheck (Netherlands): In 2000, the EyeCheck program was developed in the Netherlands as a web-based teleophthalmology program designed for primary care offices. Primary care physicians or their staff take two photos of the retina (one centered on the disc and another on the fovea), either 
dilated or undilated at the screening sites' discretion. Images are graded by an ophthalmologist. Images are designed to distinguish between no DR, non-urgent DR, and urgent DR (defined as neovascularization of the disc) [24]. As of 2010, approximately $30 \%$ of the diabetic population in the Netherlands had been screened through this program [25].

Although the system claims Category 2 validation, it has not been directly compared to ETDRS photographs or clinical examination. For validation, the founders of the program highlighted an earlier study by Van Leiden involving 442 eyes that showed moderate agreement (kappa $=0.54$ ) between two-field non mydriatic digital photography and indirect ophthalmoscopy by an ophthalmologist for the diagnosis of any level of DR [26]. A quality assessment study of the Eyecheck system showed a high inter-grader reliability $(\kappa=0.93,85 \%$ confidence interval 0.90 0.96) [24].

National health services diabetic eye screening program (United Kingdom): The NHS Diabetic Eye Screening Program (NDESP) was the first large-scale screening program instituted on a governmental level. It serves England, Wales, Scotland and Northern Ireland. [27] NDESP is reliant on local programs that adhere to the UK National Screening Committee Guidelines. In England, Wales, and Northern Ireland two mydriatic photos are taken per eye, while in Scotland, a single photo with selective mydriasis is taken [28]. The NDESP utilizes trained nonophthalmologist image graders and has built-in quality assurance controls. A primary grader determines if any diabetic retinopathy is present. If any level of diabetic retinopathy is found, the images are graded by a secondary grader blinded to the results of the first grader. Additionally, $10 \%$ of all images rated as "no retinopathy" by the first grader are screened by the secondary grader for quality assurance. When a disagreement arises, the case is adjudicated by a third grader who is an ophthalmologist, a retinal specialist, or a diabetologist with training in diabetic retinopathy image analysis $[27,29,30]$.

The NDESP grading scheme defines four levels of retinopathy (R0 to R3) and the presence or absence of CSDME (M0 or M1). Referral to a hospital eye-service is offered if level R2 retinopathy (defined by the presence of venous beading, venous loop or reduplication, intra-retinal microvascular abnormalities, multiple dot-blot hemorrhages, or a cotton wool spot) or if M1 level maculopathy is found. Urgent referral to an ophthalmologist for treatment is made if proliferative changes (Level R3) are identified [29]. Thus, the UK NDESP functions as a Category 2 program because their screening outcomes are annual telemedicine follow up for less than the R2 level disease, nonurgent referral for R2 level disease and urgent referral for R3 level disease or M1 level maculopathy.

The research branch of the NDESP, known as the UK Diabetes Research Network, cites five studies in their 2010 report as the foundation for the use of two-field mydriatic fundus photography in their tele-ophthalmology system [28]. The first study, by Taylor and colleagues, showed digital photography to be equivalent to Polaroid base film cameras [31]. A separate research group in Alberta, Canada provided evidence that stereo images of the macula were $90.6 \%$ sensitivity and $92.4 \%$ specific for detecting clinically significant DME using contact lens biomicroscopy as the reference standard in a study of 207 eyes [32]. Sharp and colleagues showed that digital photography had a $50 \%$ lower technical failure rate than color slides and was more sensitive than optometrist examination in detecting DR (83\% versus $75 \%$ respectively) [33]. Similarly, a study by Olson [34] directly compared mydriatic one-field and two-field red free digital images, color slides, and dilated fundus exam by an optometrist, to the gold standard of a dilated fundus examination by a retinal specialist in 586 patients. Both one and two-field protocols produced excellent results with a sensitivity of $93 \%$ and specificity of $87 \%$. These results were comparable to color slides and superior to optometrist examinations [34]. The final supporting literature cited by the UK Diabetes Research Network compared two-field mydriatic, non-mydriatic 1-field photography, and a gold standard of an ophthalmologist's exam. Both imaging modalities had good sensitivity (86\% mydriatic and $87.8 \%$ for non-mydriatic). Single-field non-mydriatic photography had a high technical failure rate $(19.7 \%)$ and a lower specificity $(76.7 \%$ versus $86.1 \%$ ) compared to mydriatic images [35]. With this level of evidence, the NDESP straddles the border between a Category 2 and a Category 3 system.

In 2012, nearly 2 million people were screened for DR under this program [36]. In 2013, approximately 74,000 people were referred to an eye care specialist and around 4,600 treatments were given as a result of the screening program [37]. Researchers have praised this program for lowering the rate of vision loss due to diabetes. After the United Kingdom initiated NDESP, DR was no longer the leading cause of blindness in England and Wales for the first time in five decades [38].

\section{Category 3 programs:}

Joslin Vision Network (US): The Joslin Vision Network (JVN) focuses on screening for and diagnosing DR and its protocols have undergone rigorous validation studies. The JVN system was developed in collaboration with the Veteran's Health Administration, the Department of Defense, and the Joslin Diabetes Center (Boston, MA) in 2000 [39]. JVN protocols are currently in use by the Veteran's Affairs health system, the Indian Health Services, the Department of Defense and several other private and public health systems in the US. Non-mydriatic, color, stereo photos are obtained in three fields: 1) the optic disc and macula, 2) the field nasal to the disc, and 3) the superotemporal arcade. An external non-stereo image is obtained of the anterior eye and adnexa. A trained eye-specialist reads the images manually using a software template to calculate the patient's ETDRS Severity Scale and recommends follow-up based on the disease severity [8]. The rationale behind using three-stereo fields in lieu of the standard seven-field ETDRS protocol came from earlier work using data from the Wisconsin Epidemiologic Study of Diabetic Retinopathy (WESDR). Moss and Klein [40] evaluated fundus photographs from 2,410 patients from the WESDR cohort. They found that using the three fields described above instead of the standard seven resulted in $91 \%$ agreement when looking at the 8 
ETDRS levels of DR and 95\% agreement when looking at 4 levels of DR.

One study of 535 eyes compared the JVN tele-ophthalmology diagnosis to a fundus examination by a retina specialist. There was $72.5 \%$ exact agreement and $89.3 \%$ agreement within one ETDRS level between the tele-ophthalmology diagnosis and the gold standard [8]. A second similarly designed study of 482 eyes found a sensitivity of $98 \%$ and specificity of $100 \%$ within one ETDRS grade among eyes with gradable images. Perfect agreement was reported for diagnosis of DME [41]. In a separate study of 108 diabetic eyes, moderate agreement (kappa $=0.65$, $p<0.03$ ) was achieved between tele-ophthalmology diagnosis and ETRDS seven-field color slides and high agreement (kappa $=0.85, p$-value $<0.03)$ was achieved when comparing recommended follow-up intervals [42]. Finally, a prospective study compared tele-ophthalmology images, seven-field ETDRS color photographs, and dilated fundus examination in 52 diabetic patients [43]. JVN matched the clinical examination diagnosis of CSDME and DR within 1 ETDRS level in $100 \%$ of eyes. Compared to standard seven-field ETDRS photos, the JVN diagnosis matched the diagnosis within one level of DR in $96.2 \%$ of eyes and matched in $100 \%$ of the cases of DME [43].

The JVN imaging protocol has been most widely disseminated through the Veteran's Affairs (VA) administration and the Indian Health Services (IHS). At the VA, over 120,000 tele-ophthalmology examinations have been performed using a Category 3 protocol similar to the JVN protocol. A randomized study at the VA showed patients enrolled in the tele-ophthalmology program were more likely to have annual screening than those in the traditional screening program (87\% vs. $77 \%, p<0.01)$ [44]. Studies comparing screening and laser treatment rates in the Indian Health Services, which also implemented a Category 3 protocol similar to the JVN protocol, have shown an increase in both screening and laser treatment rates by $50 \%$ in 4 years [45].

University of Alberta (Canada): In Alberta, Canada, ophthalmic telemedicine programs have been created to address the growing burden of diabetes, especially in remote regions of the province with limited access to medical care. Starting in 1997, the provincial government, in conjunction with the University of Alberta, developed a proprietary web-based system for screening and management of DR. Because many patients live over 1,000 kilometers from an ophthalmologist [9], the Alberta tele-ophthalmology network was designed to be a Category 3 program to allow for distance diagnosis and remote management when possible, referring only those who need treatment to an ophthalmologist.

The Alberta program uses seven-field ETDRS dilated digital photography with stereoscopic images of the optic disc and macula [46]. An initial study comparing this imaging modality to seven-field ETDRS photos in 241 eyes showed a perfect correlation coefficient $(r=1.00, p<0.001)$ for clinically significant DME and high-risk proliferative DR and strong correlation $(r=$ $.86, p<0.001$ ) for high-risk non-proliferative DR. [47]. A later study evaluated the effect of 16:1 image compression and remote satellite upload and found that the diagnoses were still highly accurate (kappa > 0.71) when compared to seven-field ETDRS photos [46]. As of 2009, over 6,000 patients have been screened and over 1,000 patients have been referred to an ophthalmologist for the treatment of vision-threatening retinopathy [9]. To facilitate adherence to treatment recommendations, the University of Alberta helps to arrange for all necessary testing and treatment to occur in a single visit for remote patients who screen positive for vision-threatening disease [9].

\section{Telemedicine for DR issues}

Many facets of implementation can affect the quality of a DR screening program. We will address the following issues:

- Dilation

- $\quad$ Ungradable Images

- $\quad$ Grader Training

- $\quad$ Automated Grading

- $\quad$ Access to Treatment

- Remote Education

- $\quad$ Cost-Effectiveness

Dilation: While dilation of the pupil has been shown to increase the rate of gradable images [35] and thus decrease unnecessary referrals [48], dilation increases the time of an examination and increases patient discomfort. Dilation may discourage patients and primary providers from participating in a program. Additionally, dilation has a small risk of inducing acute-closed angle glaucoma in individuals with a predisposing ocular anatomy. Acute-angle closure glaucoma is an ophthalmic emergency requiring urgent ophthalmologic care. Since the screening exams are not carried out in the presence of an ophthalmologist, the patient is at higher risk for vision loss. A large meta-analysis evaluating the risk of inducing angle-closure glaucoma found no cases in the literature when dilation with only tropicamide was used [49]. When longer acting agents were used, such a phenylephrine, rates of angle closure were between 1 in 3,380 to 1 in 20, 000 depending on the population and study methods [49]. Some programs utilize selective mydriasis, only dilating if the photographer was unable to obtain a suitable quality of non-mydriatic photo. One study found that $84 \%$ of patients with poor quality non-mydriatic photos had acceptable quality photos after dilation [50]. Overall, each program must decide whether the benefits of dilation outweigh the risks and what protocols are necessary to handle a case of acute angleclosure glaucoma should it arise.

Ungradable images: An important issue in teleophthalmology is handling un-gradable images. All un-gradable images are treated as a positive screen requiring the patient to have further, usually in-person, evaluation. Some systems rely on trained photographers or automated image analysis to immediately identify poor quality images and prompt repeat imaging. Multiple studies have found that there is a strong association between having an un-gradable image and the 
presence of ocular pathology other than DR $[8,48]$. The most common source of ocular pathology limiting the gradability of these remote images is cataract [8,51]. Therefore, referral for ungradable pathology is oftentimes appropriate.

Training of imagers: The responsibility of acquiring images is an important one for any tele-ophthalmology program. In many programs the imager not only is responsible for photography, but also assesses the quality of images and often provides basic counseling to the patient. Most programs have shifted this responsibility to non-medical staff with specific training in ophthalmic photography and diabetic retinopathy [9,39]. Often, programs have quality assurance protocols in place to ensure that individual photographers are meeting pre-specified metrics for the quality of images taken and rate of ungradable photos [7]

Training of graders: Task-shifting is a primary goal of teleophthalmology for DR as the population ages and the burden of diabetic eye disease increases [6]. Some screening programs have employed primary care providers $[52,53]$, optometrists [54] or endocrinologist [55] as graders after receiving training in reading fundus photos, but these providers are also quite timeconstrained. Other screening programs, such as the UK program and the DigiScope system (20), have trained professional "graders" who work under the guidance of an ophthalmologist.

Role of automated fundus analysis software: Automated software to grade DR is being developed to decrease the burden of reading on healthcare providers and to increase the fidelity of image grading, currently automation falls into four categories, image quality analysis, pre-screening, grading-assist, and full analysis. Quality analysis provides photographer feedback to indicate if images are of poor quality so that they can be repeated at the point-of-care $[56,57]$. Pupil size analysis can be provided to indicate when dilating the pupil would be more likely to result in a gradable image. The pre-screening software is designed to eliminate the photos without DR. The pre-screening software can reduce the number of photos that need to be graded by up to $50 \%$ [58]. Grading-assist involves lesion identification to assist with manual grading. The software will highlight potentially concerning lesions that are then confirmed by the grader [59].

Full analysis software is being developed to detect and grade the severity of any identified retinopathy. One automated detection algorithm is the Iowa Detection Program (IDP). The various iterations of IDP have been tested in several studies and show good correlation to in-person image grading. An IDP validation study of 874 patients showed a sensitivity of $96.8 \%$ and a specificity of $59.4 \%$ for identification of referable DR [60]. While the low specificity would generate unnecessary referrals, for the purpose of screening, high sensitivity is often more important to avoid missing affected patients. Other groups have tested their own fully automated analysis software in France [61] (sensitivity 92\%, specificity 50\%), the UK [62] (sensitivity 99\%, specificity 26\%), and Australia [63] (sensitivity 85\%, specificity $90 \%$ ) with the similar results. In addition to the decreasing workload and improving efficiency, an automated grading systems has the potential to decrease costs. One study estimated that incorporating automated grading software into the national screening program in Scotland would save nearly 200,000 British pounds annually [64].

Access to treatment: Screening programs must ensure whether patients received appropriate treatment to prevent loss of vision. For example, the UK national screening system is not measuring its success by the number of patients screened, but by the number of diabetics who received treatment. In rural areas and low- and middle-income countries, access to treatment represents an even larger barrier than access to screening $[65,66]$. Some networks have developed unique and innovative approaches to this problem. For instance, in the rural Indian state of Karnataka, the Nayana Project has a mobile van with diagnostic and treatment equipment. The van goes to each community to treat patients who have screened positive for disease [67]. In the screening program being developed at the Aravind Eye Institute in the state of Tamil Nadu in India, the existing community vision center infrastructure is utilized to ensure that patients receive the treatment they needed, and there is a sliding scale in place for payment for treatment [68]. The Aravind Teleophthalmology Network (ATN) utilizes remote vision centers staffed by ophthalmic technicians in addition to the conducting diabetic retinopathy camps to reach large number of patients in remote areas. Photographs are sent from the vision-centers to physicians at central grading centers and real-time consultation can be performed with video-teleconference technology [68].

Remote diabetes education: One benefit of teleophthalmology is the effect it can have on patients' general diabetic control if an educational component is included. In one study where patients underwent remote fundus photography and education, their HbA1c decreased by 1.61 points compared to patients undergoing standard endocrinology evaluation [55]. In another study, participation in the tele-ophthalmology program, which included discussion of results, an educational session, and care coordination was correlated with statistically significant improved HbA1c and low-density lipoprotein levels [69].

Cost-Effectiveness: In order for a large-scale screening program to reach enough people to be successful, it must also be cost-effective. While the value of systematic screening for DR in general has been well established $[70,71]$, the cost-effectiveness of tele-ophthalmology over traditional screening mechanisms is an area of more recent research. In one cost-effectiveness analysis of the JVN protocol compared to standard clinic-based screening, researchers predicted 148 more cases of PDR would be detected while also saving $\$ 320,000$ /year if the protocol was instituted in the Indian Health Services. Similarly, at the VA, using tele-ophthalmology would prevent 5 cases of severe vision loss while simultaneously saving $\$ 2,260,000 /$ year [72]. Another cost-effectiveness simulation modeling tele-ophthalmology versus in-person examination for the First Nation's indigenous population in Canada revealed a cost of $\$ 15,000.00 / \mathrm{QALY}$ for tele-ophthalmology compared to $\$ 37,000.00 / \mathrm{QALY}$ for traditional screening. This cost-savings would allow the program to pay off the initial equipment and hardware costs in as little as 5 years [73]. Similarly, in the Appalachian mountains of rural West 
Virginia, a tele-ophthalmology program was shown to save $\$ 150$ per patient over 7 years [74]. In areas where in-person access to an ophthalmologist is limited, DR screening through teleophthalmology can be very cost-effective.

Not all studies, however, support the cost-effectiveness of tele-ophthalmology for screening DR in all situations. In an analyzes by Kirkizlar using information from 900 VA patient charts, tele-ophthalmology was found to be cost-effective only if the system was implemented for a patient population greater than 3,500 and patients were $<80$ years old [75]. Similarly, the same analyzes that found tele-ophthalmology to be cost-effective at the VA and Indian Health Services found that it would cost an additional $\$ 13,700$ per case of severe vision loss prevented in the Department of Defense patient population [72]. Other studies have questioned the value of universal screening, either in-person or with tele-ophthalmology, in low-risk patients. Vijan found that screening low-risk diabetic patients annually versus every three years saves an average of only 3 days of sight over the course of a lifetime while it cost over $\$ 200,000 /$ QALY. They recommended either biannual screening or a customized approach for each patient [76].

\section{Conclusion}

Many tele-ophthalmology programs for DR screening have been implemented throughout the world (Table 2). Reviewing these programs through the prism of their respective ATA category allows important insight into their capabilities and goals. Category 1 programs, such as the DigiScope system, aim to screen for the presence or absence of DR and refer only those with DR for ophthalmic care, reducing the burden of screening patients without retinopathy. More advanced programs, like the Category $3 \mathrm{JVN}$ system in the US, require more extensive research prior to implementation as they are able to screen both for the presence or absence of DR and make follow-up and treatment recommendations based on the level of DR found on their remote examination.

The literature has shown that tele-ophthalmology DR screening improves screening rates, improves treatment rates, can improve general diabetes control, and can reduce vision loss due to diabetes. Additionally, screening for DR via telemedicine can be cost-effective, especially in remote and underserved areas. Given the success of these early programs, we may see a surge in tele-ophthalmology programs for diabetic screening in the future.

Table 2: Features of the diabetic retinopathy screening programs.

\begin{tabular}{|c|c|c|c|c|c|c|c|}
\hline & Ophdiat & EyePACS & Digiscope & Eye check & NDSP & JVN & University of Alberta \\
\hline Country & France & US & US & Netherlands & UK & US & Canada \\
\hline ATA Category & 1 & 1 & 1 & 2 & 2 & 3 & 3 \\
\hline Examiner & $\begin{array}{l}\text { Nurses or } \\
\text { orthoptists }\end{array}$ & $\begin{array}{l}\text { Nurse, trained } \\
\text { non-medical } \\
\text { staff }\end{array}$ & $\begin{array}{l}\text { Nurse, trained } \\
\text { non-medical } \\
\text { staff }\end{array}$ & $\begin{array}{l}\text { Nurse, trained } \\
\text { non-medical staff }\end{array}$ & $\begin{array}{l}\text { Trained non- } \\
\text { medical staff }\end{array}$ & $\begin{array}{l}\text { Trained non- } \\
\text { medical staff }\end{array}$ & $\begin{array}{l}\text { Trained non-medical } \\
\text { staff }\end{array}$ \\
\hline Setting & Hospital & $\begin{array}{l}\text { Primary care } \\
\text { office }\end{array}$ & $\begin{array}{l}\text { Primary care } \\
\text { office }\end{array}$ & $\begin{array}{l}\text { Primary care } \\
\text { office }\end{array}$ & $\begin{array}{l}\text { Primary care } \\
\text { office, hospital } \\
\text { or optician's } \\
\text { office }\end{array}$ & $\begin{array}{l}\text { Primary care } \\
\text { office or hospital }\end{array}$ & $\begin{array}{l}\text { Primary care clinic, } \\
\text { hospitals, pharmacies, } \\
\text { optometry offices }\end{array}$ \\
\hline Camera & $\begin{array}{l}\text { Cannon CR- } \\
\text { DGI Topcon } \\
\text {-NW6 }\end{array}$ & $\begin{array}{l}\text { Cannon CR-DGI } \\
\text { Cannon CR-1 }\end{array}$ & Digiscope & $\begin{array}{l}\text { Cannon CR5- } \\
\text { 45NM Topcon } \\
\text {-NW100 }\end{array}$ & Multiple models & $\begin{array}{l}\text { Topcon TRC- } \\
\text { NW6S }\end{array}$ & Multiple models \\
\hline Grader & $\begin{array}{l}\text { Ophthalmolo- } \\
\text { gist }\end{array}$ & $\begin{array}{l}\text { Optometrist or } \\
\text { ophthalmologist }\end{array}$ & $\begin{array}{l}\text { Non- } \\
\text { ophthalmologist } \\
\text { trained readers }\end{array}$ & Ophthalmologist & $\begin{array}{l}\text { Non- } \\
\text { ophthalmologist } \\
\text { trained readers }\end{array}$ & $\begin{array}{l}\text { Optometrist or } \\
\text { ophthalmologist }\end{array}$ & Ophthalmologist \\
\hline Dilation & As needed & As needed & No & As needed & Yes* & No & Yes \\
\hline $\begin{array}{l}\text { Photography } \\
\text { Scheme }\end{array}$ & $\begin{array}{l}\text { Two-non } \\
\text { stereo photos }\end{array}$ & $\begin{array}{l}\text { Three non- } \\
\text { stereo photos }\end{array}$ & $\begin{array}{l}\text { Ten non-stereo } \\
\text { photos }\end{array}$ & $\begin{array}{l}\text { Two non- stereo } \\
\text { photos }\end{array}$ & $\begin{array}{l}\text { Two stereo } \\
\text { photos** }\end{array}$ & $\begin{array}{l}\text { Three stereo } \\
\text { photos }\end{array}$ & $\begin{array}{l}\text { Two stereo and five } \\
\text { non- stereo photos }\end{array}$ \\
\hline Grading criteria & ALFEDIAM & ETDRS & ETDRS & ETDRS & $\begin{array}{l}\text { NDESP grading } \\
\text { Scheme }\end{array}$ & ETDRS & ETDRS \\
\hline Funding source & $\begin{array}{l}\text { Publically } \\
\text { funded }\end{array}$ & $\begin{array}{l}\text { For profit } \\
\text { company }\end{array}$ & $\begin{array}{l}\text { For profit } \\
\text { company }\end{array}$ & Publically funded & $\begin{array}{l}\text { Publically } \\
\text { funded }\end{array}$ & $\begin{array}{l}\text { Not for Profit- } \\
\text { organization }\end{array}$ & Publically funded \\
\hline \multicolumn{8}{|c|}{$\begin{array}{l}\text { *For England, Wales and Northern Ireland, Scotland uses selective dilation } \\
\text { **For England, Wales and Northern Ireland, Scotland uses single photo } \\
\text { ALFEDIAM: Association de Langue Francaise pours L'Etude du Diabete et des Maladies Metaboliques (French Language Association for the Study of } \\
\text { Diabetes and Metabolic Diseases) } \\
\text { ETDRS: Early Treatment of Diabetic Retinopathy Study }\end{array}$} \\
\hline
\end{tabular}




\section{References}

1. Kobrin Klein BE. Overview of epidemiologic studies of diabetic retinopathy. Ophthalmic epidemiology. 2007;14(4):179-83.

2. Group ETDRSR. Photocoagulation for diabetic macular edema Early Treatment Diabetic Retinopathy Study report number 1. Early Treatment Diabetic Retinopathy Study research group. Arch Ophthalmol. 1985;103(12): 1796-806.

3. Paz SH, Varma R, Klein R, Wu J, Azen SP, Group LALES. Noncompliance with vision care guidelines in Latinos with type 2 diabetes mellitus: the Los Angeles Latino Eye Study. Ophthalmology. 2006;113(8):1372

4. Lee PP, Feldman ZW, Ostermann J, Brown DS, Sloan FA. Longitudinal rates of annual eye examinations of persons with diabetes and chronic eye diseases. Ophthalmology. 2003; 110(10): 1952-9.

5. Huang ES, Basu A, O'Grady M, Capretta JC. Projecting Projecting the future diabetes population size and related costs for the U.S 2009;32(12):2225-9. doi: 10.2337/dc09-0459.

6. Harmon D, Merritt J. Demand for Ophthalmic Services and Ophthalmologists-A Resource Assessment. 2012.

7. Schulze-Döbold C, Erginay A, Robert N, Chabouis A, Massin P. Ophdiat $囚$ : Five-year experience of a telemedical screening programme for diabetic retinopathy in Paris and the surrounding area. Diabetes \& metabolism. 2012;38(5):450-7. doi: 10.1016/j.diabet.2012.05.003.

8. Cavallerano AA, Cavallerano JD, Katalinic P, Tolson AM, Aiello LP, Aiello LM. Use of Joslin Vision Network digital-video nonmydriatic retinal imaging to assess diabetic retinopathy in a clinical program Retina. 2003;23(2): 215-23.

9. Ng M, Nathoo N, Rudnisky CJ, Tennant MT. Improving access to eye care: teleophthalmology in Alberta, Canada. Journal of diabetes science and technology. 2009;3(2):289-96.

10. Li HK, Horton M, Bursell S-E, Cavallerano J, Zimmer-Galler I, Tennant $\mathrm{M}$, et al. Telehealth practice recommendations for diabetic retinopathy. Telemedicine and e-health. 2011;17(10):814-37. doi: 10.1089/tmj.2011.0075.

11. Chabouis A, Berdugo M, Meas T, Erginay A, Laloi-Michelin M, Jouis $\mathrm{V}$, et al. Benefits of Ophdiat $\AA$, a telemedical network to screen for diabetic retinopathy: A retrospective study in five reference hospital centres. Diabetes \& metabolism. 2009;35(3):228-32. doi: 10.1016/j. diabet.2008.12.001

12. Massin P, Erginay A, Ben Mehidi A, Vicaut E, Quentel G, Victor Z, et al. Evaluation of a new non-mydriatic digital camera for detection of diabetic retinopathy. Diabetic medicine. 2003;20(8):635-41.

13. Massin P, Chabouis A, Erginay A, Viens-Bitker C, Lecleire-Collet A, Meas T, et al. OPHDIATC: A telemedical network screening system for diabetic retinopathy in the Île-de-France. Diabetes \& metabolism. 2008;34(3):227-34.

14. Erginay A, Chabouis A, Viens-Bitker C, Robert N, Lecleire-Collet A, Massin P. OPHDIAT(C): Quality-assurance programme plan and performance of the network. Diabetes \& metabolism. 2008;34(3):23542. doi: 10.1016/j.diabet.2008.01.004.

15. Cuadros J, Bresnick G. EyePACS: an adaptable telemedicine system for diabetic retinopathy screening. Journal of diabetes science and technology. 2009;3(3):509-16.

16. Bresnick GH, Mukamel DB, Dickinson JC, Cole DR. A screening approach to the surveillance of patients with diabetes for the presence of visionthreatening retinopathy. Ophthalmology. 2000;107(1):19-24.
17. Litvin TV, Ozawa GY, Bresnick GH, Cuadros JA, Muller MS, Elsner AE, et al. Utility of Hard Exudates for the Screening of Macular Edema. Optometry \& Vision Science. 2014;91(4):370-5. doi: 10.1097/ OPX.0000000000000205.

18. Johansen MA, Fossen K, Norum J, Christoffersen T, Øritsland H, Haga D, et al. The potential of digital monochrome images versus colour slides in telescreening for diabetic retinopathy. Journal of telemedicine and telecare. 2008;14(1):27-31. doi: 10.1258/jtt.2007.060401.

19.Zeimer R, Zou S, Meeder T, Quinn K, Vitale S. A fundus camera dedicated to the screening of diabetic retinopathy in the primarycare physician's office. Investigative ophthalmology \& visual science. 2002;43(5):1581-7.

20. Zimmer-Galler I, Zeimer R. Evaluation of a reading center for detection of diabetic retinopathy with the DigiScope in primary care offices. Investigative Ophtalmology and Visual Science. 2005;46(5):1478.

21. Schiffman R, Jacobsen G, Nussbaum J, Desai U, Carey J, Glasser D, et al. Comparison of a digital retinal imaging system and seven-field stereo color fundus photography to detect diabetic retinopathy in the primary care environment. Ophthalmic Surg Lasers Imaging. 2005;36(1):46-56.

22.Zimmer-Galler IE. Diabetic Retinopathy Assessment in the Primary Care Environment: Lessons Learned from 100,000 Patient Encounters. Digital Teleretinal Screening: Springer;2012:117-26.

23.Zimmer-Galler I, Zeimer R. Results of implementation of the DigiScope for diabetic retinopathy assessment in the primary care environment Telemedicine Journal \& e-Health. 2006;12(2):89-98. doi:10.1089/ tmj.2006.12.89.

24. Abramoff MD, Suttorp-Schulten MS. Web-based screening for diabetic retinopathy in a primary care population: the EyeCheck project. Telemedicine Journal \& e-Health. 2005;11(6):668-74.

25. Lipner M. On the automated edge. EyeWorld . 2010 11/10/14;

26. van Leiden HA, Moll AC, Dekker JM, Abramoff MD, Polak BC. Photography or ophthalmoscopy for detection of diabetic retinopathy? Diabetes care. 2003;26(4):1318-1319.

27. Misra A, Bachmann M, Greenwood R, Jenkins C, Shaw A, Barakat 0 , et al. Trends in yield and effects of screening intervals during 17 years of a large UK community-based diabetic retinopathy screening programme. Diabetic medicine. 2009;26(10):1040-7. doi: 10.1111/j.1464-5491.2009.02820.x

28.2010 Annual Evidence Update on Diabetic Retinopathy. 2010 September 13, 2010. Report No.

29. Harding S, Greenwood R, Aldington S, Gibson J, Owens D, Taylor R et al. Grading and disease management in national screening for diabetic retinopathy in England and Wales. Diabetic medicine. 2003;20(12):965-71.

30. Sellahewa L, Simpson C, Maharajan P, Duffy J, Idris I. Grader agreement, and sensitivity and specificity of digital photography in a community optometry-based diabetic eye screening program. Clinical Ophthalmology (Auckland, NZ). 2014;8:345-9. doi: 10.2147/OPTH. S61483

31. Taylor D, Fisher J, Jacob J, Tooke J. The use of digital cameras in a mobile retinal screening environment. Diabetic medicine. 1999;16(8):680-6.

32. Rudnisky CJ, Hinz BJ, Tennant MT, de Leon AR, Greve MD. Highresolution stereoscopic digital fundus photography versus contact lens biomicroscopy for the detection of clinically significant macular edema. Ophthalmology. 2002;109(2):267-74. 
33. Sharp PF, Olson J, Strachan F, Hipwell J, Ludbrook A, O'donnell M, et al. The value of digital imaging in diabetic retinopathy: NCCHTA; 2003;7(30):1-119.

34. Olson J, Strachan F, Hipwell J, Goatman K, McHardy K, Forrester J, et al. A comparative evaluation of digital imaging, retinal photography and optometrist examination in screening for diabetic retinopathy. Diabetic medicine. 2003;20(7):528-34.

35. Scanlon P, Malhotra R, Thomas G, Foy C, Kirkpatrick J, Lewis-Barned $\mathrm{N}$, et al. The effectiveness of screening for diabetic retinopathy by digital imaging photography and technician ophthalmoscopy. Diabetic medicine. 2003;20(6):467-74.

36. NHS Diabetic Eye Screening Programme 2011-2012 Summary.

37. England PH. Diabetes no longer leading cause of blindness thanks to screening. 2014.

38. Liew G, Michaelides M, Bunce C. A comparison of the causes of blindness certifications in England and Wales in working age adults (16-64 years), 1999-2000 with 2009-2010. BMJ open. 2014;4(2):e004015. doi: 10.1136/bmjopen-2013-004015.

39. Conlin PR, Fisch BM, Orcutt JC, Hetrick BJ, Darkins AW. Framework for a national teleretinal imaging program to screen for diabetic retinopathy in Veterans Health Administration patients. Journal of rehabilitation research and development. 2006;43(6):741 -8.

40. Moss S, Meuer S, Klein R, Hubbard L, Brothers R, Klein B. Are seven standard photographic fields necessary for classification of diabetic retinopathy? Investigative ophthalmology \& visual science. 1989; 30(5): 823-8.

41. Ahmed J, Ward TP, Bursell S-E, Aiello LM, Cavallerano JD, Vigersky RA. The sensitivity and specificity of nonmydriatic digital stereoscopic retinal imaging in detecting diabetic retinopathy. Diabetes care. 2006;29(10):2205-9.

42. Bursell S-E, Cavallerano JD, Cavallerano AA, Clermont AC, BirkmirePeters D, Aiello LP, et al. Stereo nonmydriatic digital-video color retinal imaging compared with Early Treatment Diabetic Retinopathy Study seven standard field 35-mm stereo color photos for determining level of diabetic retinopathy. Ophthalmology. 2001;108(3):572-85.

43. Cavallerano JD, Aiello LP, Cavallerano AA, Katalinic P, Hock K, Kirby R, et al. Nonmydriatic digital imaging alternative for annual retinal examination in persons with previously documented no or mild diabetic retinopathy. American journal of ophthalmology. 2005;140(4):667. e1-. e8.

44. Conlin PR, Fisch BM, Cavallerano AA, Cavallerano JD, Bursell S, Aiello LM. Nonmydriatic teleretinal imaging improves adherence to annual eye examinations in patients with diabetes. Journal of rehabilitation research and development. 2006;43(6):733.

45. Wilson C, Horton M, Cavallerano J, Aiello LM. Addition of primary carebased retinal imaging technology to an existing eye care professional referral program increased the rate of surveillance and treatment of diabetic retinopathy. Diabetes care. 2005; 28(2): 318-22.

46. Rudnisky CJ, Tennant MT, Weis E, Ting A, Hinz BJ, Greve MD. Webbased grading of compressed stereoscopic digital photography versus standard slide film photography for the diagnosis of diabetic retinopathy. Ophthalmology. 2007;114(9):1748-54.

47. Tennant MT, Greve MD, Rudnisky CJ, Hillson TR, Hinz BJ. Identification of diabetic retinopathy by stereoscopic digital imaging via teleophthalmology: a comparison to slide film. Canadian Journal of Ophthalmology/Journal Canadien d'Ophtalmologie. 2001;36(4):18796.
48. Baeza M, Orozco-Beltrán D, Gil-Guillen V, Pedrera V, Ribera M, Pertusa $S$, et al. Screening for sight threatening diabetic retinopathy using nonmydriatic retinal camera in a primary care setting: to dilate or not to dilate? International journal of clinical practice. 2009;63(3):433-8. doi: 10.1111/j.1742-1241.2008.01921.x.

49. Pandit R, Taylor R. Mydriasis and glaucoma: exploding the myth. A systematic review. Diabetic medicine. 2000;17(10):693-9.

50. Pugh JA, Jacobson JM, Van Heuven W, Watters JA, Tuley MR, Lairson DR, et al. Screening for diabetic retinopathy: the wide-angle retinal camera. Diabetes care. 1993;16(6): 889-95.

51. Cavallerano AA, Cavallerano JD, Katalinic P, Blake B, Rynne M, Conlin $\mathrm{PR}$, et al. A telemedicine program for diabetic retinopathy in a Veterans Affairs Medical Center-the Joslin Vision Network eye health care model. American journal of ophthalmology. 2005139(4):597604.

52. Castro AF, Silva-Turnes JC, Gonzalez F. Evaluation of retinal digital images by a general practitioner. Telemedicine and e-health. 2007;13(3):287-92.

53. Andonegui J, Serrano L, Eguzkiza A, Berástegui L, Jiménez-Lasanta L, Aliseda D, et al. Diabetic retinopathy screening using teleophthalmology in a primary care setting. Journal of telemedicine and telecare. 2010;16(8): 429-32. doi: 10.1258/jtt.2010.091204.

54. Hulme S, Tin-U A, Hardy K, Joyce P. Evaluation of a district-wide screening programme for diabetic retinopathy utilizing trained optometrists using slit-lamp and Volk lenses. Diabetic medicine. 2002;19(9):741-5.

55. Salti H, Cavallerano JD, Salti N, Jawhari DJ, Haddad S, Sun JK, et al. Nonmydriatic retinal image review at time of endocrinology visit results in short-term HbA1c reduction in poorly controlled patients with diabetic retinopathy. Telemedicine and e-health. 2011;17(6):4159. doi: $10.1089 / \mathrm{tmj} .2010 .0180$

56. Niemeijer M, Abramoff MD, van Ginneken B. Image structure clustering for image quality verification of color retina images in diabetic retinopathy screening. Medical image analysis. 2006; 10(6): 888-98.

57. Fleming AD, Philip S, Goatman KA, Olson JA, Sharp PF. Automated assessment of diabetic retinal image quality based on clarity and field definition. Investigative ophthalmology \& visual science. 2006;47(3):1120-5.

58. Hipwell J, Strachan F, Olson J, McHardy K, Sharp P, Forrester J. Automated detection of microaneurysms in digital red-free photographs: a diabetic retinopathy screening tool. Diabetic medicine. 2000;17(8):588-94.

59. Joshi GD, Sivaswamy J. DrishtiCare: a telescreening platform for diabetic retinopathy powered with fundus image analysis. Journal of diabetes science and technology. 2011;5(1):23-31.

60. Abràmoff MD, Folk JC, Han DP, Walker JD, Williams DF, Russell SR, et al. Automated analysis of retinal images for detection of referable diabetic retinopathy. JAMA ophthalmology. 2013;131(3):351-7. doi: 10.1001/jamaophthalmol.2013.1743.

61. Dupas B, Walter T, Erginay A, Ordonez R, Deb-Joardar N, Gain P, et al. Evaluation of automated fundus photograph analysis algorithms for detecting microaneurysms, haemorrhages and exudates, and of a computer-assisted diagnostic system for grading diabetic retinopathy. Diabetes \& metabolism. 2010;36(3):213-20. doi: 10.1016/j. diabet.2010.01.002.

62. Goatman K, Charnley A, Webster L, Nussey S. Assessment of automated 
disease detection in diabetic retinopathy screening using two-field photography. PLOS one. 2011;6(12): e27524. doi: 10.1371/journal. pone. 0027524

63. Jelinek HJ, Cree MJ, Worsley D, Luckie A, Nixon P. An automated microaneurysm detector as a tool for identification of diabetic retinopathy in rural optometric practice. Clinical and Experimental Optometry. 2006;89(5):299-305.

64. Scotland GS, McNamee P, Philip S, Fleming AD, Goatman KA, Prescott GJ, et al. Cost-effectiveness of implementing automated grading within the national screening programme for diabetic retinopathy in Scotland. British Journal of Ophthalmology. 2007;91(11):1518-23.

65. Hartnett M, Key IJ, Loyacano NM, Horswell RL, DeSalvo KB. Perceived barriers to diabetic eye care: Qualitative study of patients and physicians. Archives of ophthalmology. 2005;123(3):387-91.

66. Wylie-Rosett J, Basch C, Walker EA, Zybert P, Shamoon H, Engel S, et al. Ophthalmic referral rates for patients with diabetes in primarycare clinics located in disadvantaged urban communities. Journal of Diabetes and its Complications. 1995;9(1):49-54.

67. Murthy KR, Murthy PR, Rao S, Murthy GJ, Kapur A, Lefebvre P. A novel model to deliver advanced eye care for people with diabetes living in resource-poor settings: results of care provided to date. Diabetes care. 2012;35(4):e31-e. doi: 10.2337/dc11-2098.

68. Bai VT, Murali V, Kim R, Srivatsa S. Teleophthalmology-based rural eye care in India. Telemedicine and e-health. 2007;13(3):313-21.

69. Fonda SJ, Bursell S-E, Lewis DG, Garren J, Hock K, Cavallerano J. The relationship of a diabetes telehealth eye care program to standard eye care and change in diabetes health outcomes. Telemedicine and e-health. 2007;13(6):635-44.

70. Javitt JC, Aiello LP. Cost-effectiveness of detecting and treating diabetic retinopathy. Annals of internal medicine. 1996;124(1_Part_2):164-9.

71. Jones S, Edwards R. Diabetic retinopathy screening: a systematic review of the economic evidence. Diabetic medicine. 2010;27(3): 24956. doi: 10.1111/j.1464-5491.2009.02870.x.

72. Whited JD, Datta SK, Aiello LM, Aiello LP, Cavallerano JD, Conlin PR, et al. A modeled economic analysis of a digital teleophthalmology system as used by three federal healthcare agencies for detecting proliferative diabetic retinopathy. Telemedicine Journal \& e-Health. 2005;11(6):641-51.

73. Maberley D, Walker H, Koushik A, Cruess A. Screening for diabetic retinopathy in James Bay, Ontario: a cost-effectiveness analysis. Canadian Medical Association Journal. 2003;168(2):160-4.

74. Richardson D, Fry R, Krasnow M. Cost-savings analysis of telemedicine use for ophthalmic screening in a rural Appalachian health clinic. The West Virginia medical journal. 2012;109(4):52-5.

75. Kirkizlar E, Serban N, Sisson JA, Swann JL, Barnes CS, Williams MD. Evaluation of Telemedicine for Screening of Diabetic Retinopathy in the Veterans Health Administration. Ophthalmology. 2013;120(12):260410. doi: 10.1016/j.ophtha.2013.06.029.

76. Vijan S, Hofer TP, Hayward RA. Cost-utility analysis of screening intervals for diabetic retinopathy in patients with type 2 diabetes mellitus. Jama. 2000;283(7):889-96. 